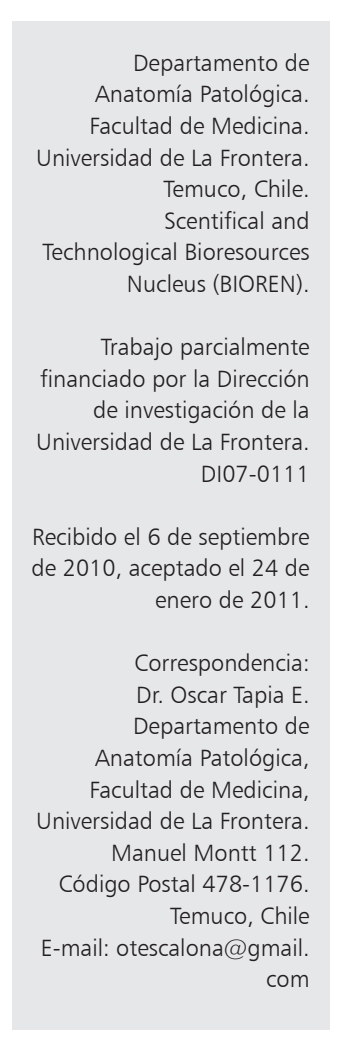

\section{Cáncer gástrico sin compromiso ganglionar linfático (pNo): características demográfico-patológicas y factores pronósticos}

OSCAR TAPIA E., MIGUEL VILLASECA H., ENRIQUE BELLOLIO J., JUAN CARLOS ARAYA O., JUAN CARLOS ROA S.

\section{Prognostic factors for gastric cancer without lymph node involvement}

Background: The absence of lymph node involvement (NO) in gastric cancer is associated with a better survival. However some NO gastric tumors still have a bad prognosis. Aim: To study demographic and morphological variables associated with prognosis in No gastric carcinoma. Material and Methods: Review of pathological records of a regional general hospital, identifying patients with a NO gastric cancer surgically excised between 1986 and 2003. Results: In the study period, 459 gastrectomies were performed for gastric cancer and in $32 \%$, the tumor was devoid of lymph node involvement. These later patients were followed for a median of 64 months with a $71 \%$ five years actuarial survival. Bivariate analysis identified age, tumor size, gastric wall infiltration, pathological type according to Lauren and Ming, lymphovascular involvement, number of lymph nodes excised and TNM stage as prognostic values. Multivariate analysis disclosed the level of gastric wall infiltration, the presence of a poorly differentiated tumor, lymphatic vascular involvement, number of excise lymph nodes and tumor size as independent prognostic factors. Conclusions: NO gastric tumors are found in 32\% of gastrectomies for gastric cancer and have a $71 \%$ five years actuarial survival. Gastric wall infiltration, pathological degree of differentiation, tumor size and lymphovascular involvement are independent prognostic factors.

(Rev Med Chile 2011; 139: 432-438).

Key words: Gastrectomy; Risk factors; Stomach neoplasms; Survival.
S e encuentra ampliamente documentado en la literatura que el nivel de infiltración tumoral en la pared gástrica ( $\mathrm{pT}$ ) y compromiso ganglionar linfático $(\mathrm{pN})$ son de los factores pronósticos independientes más importantes en la supervivencia (SV) de pacientes operados por cáncer gástrico (CG). De esta manera, en sujetos sin compromiso ganglionar linfático ( $\mathrm{pNO}$ ) la probabilidad de recurrencia es significativamente menor que en pacientes con metástasis linfáticas $(\mathrm{N}+)$, mientras que la supervivencia global es significativamente mayor en pacientes $\mathrm{N}^{1-7}$.
Sin embargo, a pesar de estas consideraciones, en algunos casos de CG pN0 el tumor presentará un comportamiento biológico distinto, con tendencia a la recurrencia local y pobre pronóstico. Con la finalidad de explicar este errático comportamiento de algunos carcinomas gástricos $\mathrm{pN} 0$, autores han realizado estudios enfocados a la identificación de variables clínicas y morfológicas asociadas al pronóstico de pacientes resecados por CG pN0 con intención curativa (R0), demostrando ellos entre los principales factores relacionados al pronóstico el tamaño tumoral, nivel de infiltra- 
Factores pronósticos en cáncer gástrico pNO - O. Tapia et al

ción parietal, grado de diferenciación histológico e invasión linfovascular, entre otros; mientras que los resultados son contradictorios en cuanto a la importancia pronóstica de la edad, género, localización tumoral y tipo de resección efectuada

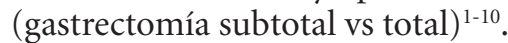

El objetivo de este estudio fue comparar las características clínico-patológicas de pacientes con CG pN0 y N+ junto con determinar la existencia de asociación de estas variables con la SV de pacientes resecados por CG pN0.

\section{Material y Método}

\section{Diseño del estudio}

Estudio de cohorte histórica o retrospectiva.

\section{Marco}

Se recogieron los datos provenientes de pacientes diagnosticados de CG en la Unidad de Anatomía Patológica del Hospital Hernán Henríquez Aravena de la ciudad de Temuco entre enero de 1986 y diciembre de 2003.

\section{Pacientes}

Se incluyeron todos los casos de gastrectomías por CG diagnosticados de forma consecutiva en el período e institución antes señalados, en los cuales se realizó únicamente tratamiento quirúrgico con disección ganglionar extendida (D2). El seguimiento de los casos se realizó mediante el estudio de las historias clínicas y certificados de defunción obtenidos del Registro Civil e Identificación.

\section{Criterios de exclusión}

Fueron excluidos aquellos sujetos sin indicación quirúrgica por enfermedad avanzada o condiciones clínicas del paciente, casos sometidos sólo a laparotomía exploradora por enfermedad diseminada, pacientes sometidos a cirugías paliativas y casos que recibieron tratamientos adicionales a la cirugía (27 pacientes). Se excluyeron del análisis de SV pacientes que fallecieron en los 30 días posteriores a la cirugía, considerados como mortalidad perioperatoria (Figura 1).

\section{Protocolo de procesamiento}

En este período, el procesamiento de las piezas quirúrgicas de gastrectomía fue realizado en forma uniforme a través de la apertura, extensión y fija- ción de la pieza quirúrgica en planchas de parafina sólida, con un examen macroscópico diferido. El examen microscópico consideró el estudio del tumor, ganglios linfáticos y ambos bordes de sección quirúrgicos (proximal y distal). Tumores avanzados fueron examinados mediante múltiples secciones representativas mientras que lesiones incipientes fueron muestreadas en su totalidad con el objeto de determinar el máximo nivel de infiltración en la pared gástrica.

\section{Examen ganglionar linfático}

La búsqueda de ganglios linfáticos fue realizada con la muestra en fresco, identificándose los respectivos grupos ganglionares y posterior fijación en formalina. Todos lo ganglios linfáticos fueron seccionados en su eje mayor, seleccionándose una muestra representativa y teñidos con hematoxilina-eosina.

\section{Variables de interés}

De acuerdo al pN se obtuvieron los siguientes grupos: 1) Pacientes sin metástasis ganglionar (pN0) y 2) Pacientes con metástasis ganglionar $(\mathrm{N}+)$. La variable resultado fue SV, medida en meses posterior a la cirugía.

\section{Plan de análisis}

Utilizando los paquetes estadísticos Epi-info 6.0 y Stata 9.0, se realizó un análisis exploratorio de los datos, lo que determinó la necesidad de categorizar algunas variables continuas según su distribución: Edad (menores de 65 años e igual o mayores de 65 años), tamaño tumoral (menores de $40 \mathrm{~mm}$ e igual o mayores de $40 \mathrm{~mm}$ ) considerando para su categorización el eje longitudinal mayor, localización tumoral (categorizada en tres

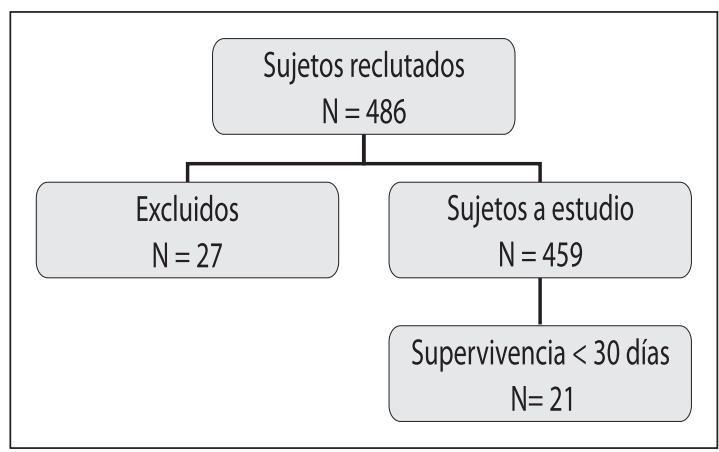

Figura 1. 
tercios: superior [cardial y subcardial], medio [cuerpo] e inferior [antral y pilórico)] acorde con las normas de la sociedad japonesa (en aquellos casos de tumores con compromiso de más de un segmento se categorizaron como difusos), tipo macroscópico (categorizada según la clasificación macroscópica de la sociedad japonesa de gastroenterología endoscópica para tumores incipientes y según la clasificación de Bormann para tumores avanzados), nivel de infiltración (categorizado según el estadio pT de la $77^{\circ}$ edición de la American Joint Committee of Cancer (AJCC) para CG), grado de diferenciación histológica (categorizado en bien, moderado y poco diferenciado), prevaleciendo para su categorización el menor grado de diferenciación presente en el tumor, compromiso ganglionar linfático (dicotomizado en positivos $[\mathrm{N}+]$ o negativos [pN0]), tipo de resección quirúrgica (gastrectomía subtotal y total), número de ganglios linfáticos resecados (categorizados en menos de 15, 15-30 y más de 30 ganglios linfáticos resecados) y estadio TNM de acuerdo a la $7^{\circ}$ edición AJCC para CG. Variables morfológicas tales como tipo histológico según Lauren y Ming fueron clasificados en base a las definiciones ya establecidas en la literatura. Para el análisis multivariado grado de diferenciación fue categorizado en tumores bien-moderadamente y poco diferenciados mientras que para nivel de infiltración en pT1-pT2 y pT3-pT4.

\section{Sesgos}

Se minimizó el sesgo de clasificación mediante la comprobación de los datos por investigadores independientes (OTE, JCR, MV y JCA).

\section{Tamaño de la muestra}

No se realizó estimación de tamaño de la muestra pues se trabajó con la totalidad del universo de pacientes con CG sometidos a gastrectomía en el período e institución antes señalados.

\section{Métodos estadísticos}

Se aplicó estadística descriptiva con cálculo de medidas de tendencia central y extrema; y estadística analítica, aplicando $\chi^{2}$ de Pearson y exacto de Fisher para comparación de variables categóricas; T-Student para comparación de promedios en variables continuas; confección de curvas de SV de Kaplan-Meier y ulterior comparación de estas por medio de la aplicación de Log-rank test. Finalmen- te, se realizaron modelos de regresión logística para determinación de fuerza de asociación con cálculo de odds ratio (OR) y sus respectivos intervalos de confianza de 95\% (IC 95\%).

\section{Resultados}

De acuerdo con los criterios de inclusión, en el período antes señalado se realizaron 459 gastrectomías por CG; de las cuales se verificaron 147 casos (32\%) de CG pN0 y 312 casos (68\%) de CG N+.

El promedio de edad para CG pN0 y N+ fue $60,5 \pm 11,9$ y $59,8 \pm 11,9$ años respectivamente $(\mathrm{p}=\mathrm{NS})$. Para los casos pN0, 65\% (96) de los pacientes eran de género masculino, sin verificarse diferencias estadísticas significativas con el grupo $\mathrm{N}+$ (Tabla 1). El promedio de ganglios linfáticos resecados para tumores $\mathrm{pN} 0$ y $\mathrm{N}+$ fue 27,4 y 32,3 ganglios respectivamente $(\mathrm{p}=\mathrm{NS})$.

Al comparar ambos grupos se constataron diferencias estadísticamente significativas en las variables tamaño tumoral $(<40 \mathrm{~mm})$, grado de diferenciación histológico (tumores bien diferenciados), tipo histológico según Lauren (variante intestinal) y Ming (variante expansiva), nivel de infiltración (pT1-pT2) y estadio TNM (estadio I y II) en tumores N- (Tabla 1).

La mediana de seguimiento fue de 64 meses (1 a 206) y 23 meses (1 a 194) para el grupo de CG pN0 y CG N+ respectivamente ( $\mathrm{p}<0,0001)$. La SV global actuarial observada a 5 y 10 años para tumores pN0 fue $71 \%$ y $61 \%$ respectivamente, mientras que para tumores $\mathrm{N}+$ fue de $33 \%$ y $30 \%$ a 5 y 10 años respectivamente.

Al aplicar análisis bivariados en el grupo de CG pN0 se constató asociación entre SV para las variables detalladas en la Tabla 2: grupo etario $(\mathrm{p}=0,002)$, tamaño tumoral $(\mathrm{p}=0,01)$, nivel de infiltración $(\mathrm{p}=0,0001)$, grado de diferenciación histológico $(\mathrm{p}=0,03)$, tipo histológico según Lauren $(\mathrm{p}=0,04)$ y Ming $(\mathrm{p}=0,001)$, compromiso linfovascular $(\mathrm{p}=0,001)$, número de ganglios linfáticos resecados $(\mathrm{p}<0,0001)$ y estadio TNM $(\mathrm{p}<$ 0,0001 ). El análisis multivariado permitió verificar como variables asociadas a la SV en pacientes con CG pN0 al nivel de infiltración tumoral, grado de diferenciación en su variante poco diferenciada, recuento $<15$ ganglios resecados, compromiso vascular linfático y tamaño tumoral $\geq 40 \mathrm{~mm}$. (Tabla 3). 
Factores pronósticos en cáncer gástrico pNO - O. Tapia et al

Tabla 1. Distribución de variables clínico-morfológicas según nivel estado ganglionar linfático

\begin{tabular}{|c|c|c|c|}
\hline Variables & $\begin{array}{l}\text { CG pNO } \\
(n=147)\end{array}$ & $\begin{array}{c}\text { CG N+ } \\
(n=312)\end{array}$ & $\mathbf{p}$ \\
\hline Género \% (n) & & & 0,32 \\
\hline Femenino & $35,0(51)$ & $30,0 \quad(94)$ & \\
\hline Masculino & $65,0(96)$ & $70,0(218)$ & \\
\hline Resección quirúrgica \% (n) & & & 0,35 \\
\hline Gastrectomía subtotal & $52,0(76)$ & $47,0(147)$ & \\
\hline Gastrectomía total & $48,0(71)$ & $53,0(165)$ & \\
\hline \multicolumn{4}{|l|}{ Localización \% (n) } \\
\hline $1 / 3$ superior & $30,0(44)$ & $30,0 \quad(94)$ & \\
\hline $1 / 3$ medio & $36,0(53)$ & $24,0 \quad(75)$ & \\
\hline $1 / 3$ inferior & $34,0(50)$ & $43,0(134)$ & \\
\hline Difuso & $0,0 \quad(0)$ & $3,0 \quad(9)$ & \\
\hline Nivel de infiltración \% (n) & & & $<0,0001$ \\
\hline CG incipiente & $39,0(57)$ & $3,0 \quad(9)$ & \\
\hline CG avanzado & $61,0(90)$ & $97,0(303)$ & \\
\hline Tamaño tumoral \% (n) & & & $<0,0001$ \\
\hline$<40 \mathrm{~mm}$ & $46,0(68)$ & $17,0(53)$ & \\
\hline$\geq 40 \mathrm{~mm}$ & $54,0(79)$ & $83,0(259)$ & \\
\hline Grado de Diferenciación \% (n) & & & $<0,0001$ \\
\hline Bien diferenciado & $22,0(32)$ & $2,0 \quad(6)$ & \\
\hline Moderadamente diferenciado & $34,0(50)$ & $32,0(100)$ & \\
\hline Poco diferenciado & $44,0(64)$ & $66,0(206)$ & \\
\hline Tipo histológico según Lauren \% (n) & & & $<, 00001$ \\
\hline Intestinal & $52,0(76)$ & $30,0 \quad(94)$ & \\
\hline Difuso & $48,0(71)$ & $70,0(218)$ & \\
\hline Tipo histológico según Ming \% (n) & & & $<, 00001$ \\
\hline Expansivo & $63,0(93)$ & $40,0(125)$ & \\
\hline Infiltrativo & $37,0(54)$ & $60,0(187)$ & \\
\hline Estadio pT \% (n) & & & $<0,0001$ \\
\hline pT1-pT2 & $56,0(82)$ & 10,0 & \\
\hline рT3-рT4 & $44,0(65)$ & $90,0(280)$ & \\
\hline Estadio TNM \% (n) & & & $<0,0001$ \\
\hline I & $55,0(81)$ & $1,0 \quad(3)$ & \\
\hline ॥ & $26,0(38)$ & 17,0 & \\
\hline III & $19,0(28)$ & $74,0(232)$ & \\
\hline IV & $0,0(0)$ & $8,0(24)$ & \\
\hline
\end{tabular}


Tabla 2. Análisis univariado de variables clínico-morfológicas pronósticas en pacientes con cáncer gástrico pNO

\begin{tabular}{|c|c|c|}
\hline Variables & $\begin{array}{c}\text { SV } 5 \text { años* } \\
\%\end{array}$ & $\mathbf{p}$ \\
\hline $\begin{array}{l}\text { Género } \\
\text { Femenino } \\
\text { Masculino }\end{array}$ & $\begin{array}{l}76,5 \\
71,6\end{array}$ & 0,67 \\
\hline $\begin{array}{l}\text { Grupo etario } \\
\quad<65 \text { años } \\
\geq 65 \text { años }\end{array}$ & $\begin{array}{l}80,1 \\
63,0\end{array}$ & 0,002 \\
\hline $\begin{array}{l}\text { Resección quirúrgica } \\
\text { Gastrectomía subtotal } \\
\text { Gastrectomía total }\end{array}$ & $\begin{array}{l}77,3 \\
69,1\end{array}$ & 0,17 \\
\hline $\begin{array}{l}\text { Localización } \\
1 / 3 \text { superior } \\
1 / 3 \text { medio } \\
1 / 3 \text { inferior } \\
\text { Difuso }\end{array}$ & $\begin{array}{r}75,7 \\
78,7 \\
67,0 \\
3,0\end{array}$ & 0,24 \\
\hline $\begin{array}{l}\text { Tamaño tumoral } \\
\quad \leq 40 \mathrm{~mm} \\
\quad>40 \mathrm{~mm}\end{array}$ & $\begin{array}{l}81,9 \\
65,0\end{array}$ & 0,01 \\
\hline $\begin{array}{l}\text { Grado de Diferenciación } \\
\text { Bien diferenciado } \\
\text { Moderadamente diferenciado } \\
\text { Poco diferenciado }\end{array}$ & $\begin{array}{l}89,7 \\
74,6 \\
65,0\end{array}$ & 0,03 \\
\hline $\begin{array}{l}\text { Tipo histológico según Lauren } \\
\text { Intestinal } \\
\text { Difuso }\end{array}$ & $\begin{array}{l}55,4 \\
41,2\end{array}$ & 0,04 \\
\hline $\begin{array}{l}\text { Tipo histológico según Ming } \\
\text { Expansivo } \\
\text { Infiltrativo }\end{array}$ & $\begin{array}{l}49,2 \\
30,9\end{array}$ & 0,001 \\
\hline $\begin{array}{l}\text { Nivel de infiltración } \\
\text { pT1 } \\
\text { pT2 } \\
\text { pT3 } \\
\text { pT4 }\end{array}$ & $\begin{array}{l}86,4 \\
81,4 \\
62,6 \\
34,5\end{array}$ & 0,0001 \\
\hline $\begin{array}{l}\text { Número ganglios resecados } \\
<15 \text { ganglios linfáticos } \\
\text { 15-30 ganglios linfáticos } \\
>31 \text { ganglios linfáticos }\end{array}$ & $\begin{array}{l}37,5 \\
69,0 \\
76,7\end{array}$ & $<0,0001$ \\
\hline $\begin{array}{l}\text { Estadio TNM } \\
\text { I } \\
\text { II } \\
\text { III } \\
\text { IV }\end{array}$ & $\begin{array}{l}87,0 \\
62,1 \\
44,5 \\
-\end{array}$ & $<0,0001$ \\
\hline $\begin{array}{l}\text { Compromiso linfovascular } \\
\text { No } \\
\text { Sí }\end{array}$ & $\begin{array}{l}78,1 \\
57,7\end{array}$ & 0,001 \\
\hline $\begin{array}{l}\text { Compromiso neural } \\
\text { No } \\
\text { Si }\end{array}$ & $\begin{array}{l}76,9 \\
72,5\end{array}$ & 0,73 \\
\hline
\end{tabular}

* Supervivencia actuarial a 5 años.

\section{Discusión}

Se encuentra ampliamente documentado en la literatura el impacto pronóstico del compromiso ganglionar linfático tanto en la recurrencia como SV de pacientes resecados por CG, presentando pacientes con CG N+ mayor tasa de recurrencia y menor SV que pacientes $\mathrm{pN} 0$, verificando en nuestra serie una SV actuarial global a 5 años para tumores pN0 y N+ de $71 \%$ y $33 \%$ respectivamente $(\mathrm{p}<0,0001)^{1-7,11}$. La SV reportada a 5 años para pacientes con CG pN0 alcanza hasta 90\%, describiendo algunos autores que estos casos tendrían características clínico-morfológicas y un comportamiento biológico similar a tumores gástricos incipientes; es por ello que la identificación de variables pronósticas en pacientes con CG pN0 sometidos a cirugía curativa (R0) adquiere vital importancia, de manera de identificar a aquellos pacientes que presentaran un comportamiento biológico distinto al esperado y que por lo tanto, se beneficiarían de terapias complementarias a la cirugía ${ }^{16,12}$. En este sentido, trabajos han identificado a variables como el tamaño tumoral, nivel de infiltración parietal, grado de diferenciación histológico, invasión linfovascular y tipo de resección asociados a la SV y recurrencia de la enfermedad ${ }^{1-5,7-10}$.

Al igual que lo publicado por otros autores, en nuestra serie el análisis bivariado para el grupo de pacientes con CG pN0 demostró como factores asociados a peor SV el tamaño tumoral ( $\geq 40 \mathrm{~mm}$ ), grupo etario $\geq$ de 65 años, nivel de infiltración tumoral en la pared gástrica (pT3-pT4), grado de diferenciación histológico en su categoría poco diferenciado, tipo histológico según Lauren (variante difuso) y Ming (variante infiltrativo), presencia de compromiso linfovascular y estadio $\mathrm{TNM}^{6,11,13-16}$. Por el contrario, no encontramos asociación estadística significativa con SV de acuerdo a la localización tumoral y tipo de resección quirúrgica realizada; esto último probablemente 
Factores pronósticos en cáncer gástrico pNO - O. Tapia et al

Tabla 3. Análisis multivariado

\begin{tabular}{|llcc|}
\hline Variable & OR & IC 95\% & p \\
\hline Edad $\geq 65$ años & 0,96 & $0,07-12,5$ & 0,97 \\
\hline Género masculino & 1,21 & $0,07-18,5$ & 0,88 \\
\hline Gastrectomía subtotal & 4,63 & $0,18-115,1$ & 0,34 \\
\hline Tumor $>40 \mathrm{~mm}$ & 1,89 & $1,41-2,54$ & 0,01 \\
\hline Tumor difuso de Lauren & 2,09 & $0,01-405,4$ & 0,28 \\
\hline Tumor poco diferenciado & 2,23 & $1,32-4,27$ & 0,003 \\
\hline$<15$ ganglios linfáticos resecados & 3,4 & $1,48-5,89$ & 0,001 \\
\hline Infiltración tumoral parietal & 6,48 & $2,52-10,21$ & $<0,0001$ \\
\hline Compromiso vascular linfático & 1,76 & $1,28-2,84$ & 0,01 \\
\hline
\end{tabular}

explicado por la heterogeneidad muestral producto de la inclusión en otras series tanto de sujetos con y sin disección ganglionar extendida (D2) producto del tipo de cirugía realizada según la localización tumoral, resultando por lo tanto, una proporción de ellos con un número de ganglios linfáticos examinados menos que óptimo para una adecuada etapificación $\mathrm{pN}$ y el consiguiente sesgo en la categorización ${ }^{11,13,17}$.

La importancia pronóstica como factor independiente del tamaño tumoral es todavía controversial, por un lado algunos autores demuestran en sus trabajos que esta variable se encuentra estrechamente relacionada con los 2 principales factores pronósticos independientes en CG: nivel de infiltración y metástasis linfática, por lo que carece de valor pronóstico independiente; mientras que por otro lado, Kim et al demostraron en su estudio multivariado de pacientes con CG pN0 la importancia pronóstica del tamaño tumoral $(\geq 50$ $\mathrm{mm}$ ) presentando un riesgo aumentado de hasta 1,5 veces comparado con tumores menores de 50 $\mathrm{mm}$. Del mismo modo, en nuestra serie, tumores $\geq 40 \mathrm{~mm}$ presentaron un OR de 1,89 (IC 95\%: $1,41-2,54 ; \mathrm{p}=0,01)^{6,11,13,16}$.

El compromiso linfovascular se demostró en el $23 \%$ de los CG pN0, fluctuando su presencia entre $10 \%$ y $33 \%$ en los distintos trabajos publicados; esta resultó ser un importante predictor pronóstico independiente en sujetos con tumores $\mathrm{pN} 0$, presentando los sujetos con compromiso linfovascular hasta 1,76 veces mayor riesgo de fallecer comparados con los CG pN0 que no presentaban compromiso linfovascular (IC 95\%: 1,2-2,8; $\mathrm{p}=$ 0,01 ), al igual que lo reportado por Lee et $\mathrm{al}^{6,9,13,17}$.
Por otro lado, si bien se ha demostrado el impacto pronóstico del compromiso tumoral perineural en neoplasias malignas de otras localizaciones, en CG la información es escasa y controversial; verificando en nuestro serie su presencia en $12 \%$ de los casos y careciendo de valor pronóstico en el análisis multivariado, al igual que lo reportado por Kooby et al ${ }^{13,18-22}$.

Como se ha mencionado, el nivel de infiltración tumoral en la pared gástrica (pT) es otro de los predictores pronósticos más relevantes en pacientes resecados por cáncer gástrico (CG); verificando en nuestro grupo que el nivel de infiltración tumoral permanece como una de las principales variables pronósticas para sujetos con CG N-, presentando un OR de 6,48 (IC 95\%: 2,52$10,21 ; \mathrm{p}<0,0001)$.

Finalmente, es importante mencionar dos elementos trascendentales al momento de realizar la etapificación según compromiso ganglionar: primero, se requiere de un adecuado recuento ganglionar que permita evitar etapificar incorrectamente a un paciente como pNO debido al examen de un número insuficiente o no representativo de ganglios linfáticos, hecho que se demuestra en este estudio en el analisis bi y multivariado, al observar una menor SV en el grupo de pacientes con recuento de ganglios resecados $<15$, explicado por incluir este grupo con alta probabilidad falsos pN0 atribuibles al número insuficiente de ganglios examinados; y segundo, el cuidadoso examen histopatológico de los linfonodos que le permita al anatomo-patólogo concluir que los ganglios linfáticos no están comprometidos debiendo para ello realizar cortes histológicos, técnicas histoquí- 
micas o de inmunohistoquímica adicionales según corresponda en cada caso. En nuestro trabajo el promedio de ganglios linfáticos resecados para tumores pN0 fue 27,4 con un rango de 11-68 ganglios linfáticos; realizándose tinciones con hematoxilina-eosina de rutina en todos los casos, cortes adicionales y tinciones histoquímicas especiales para mucinas (PAS y azul alcián) en forma aislada y no siendo necesario realizar técnicas de inmunohistoquímica.

En base a lo anteriormente expuesto, podemos concluir que se verificó $32 \%$ de CG pN0, los que presentan una SV de $71 \%$ a 5 años. Para este grupo en estudio, el nivel de infiltración tumoral, el tamaño tumoral, el compromiso linfovascular y el grado de diferenciación tumoral resultaron factores independientes asociados a la SV.

\section{Referencias}

1. Bruno L, Nesi G, Montinaro F, Carassale G, Boddi V, Bechi $\mathrm{P}$, et al. Clinicopathologic characteristics and outcome indicators in node-negative gastric cancer. J Surg Oncol 2000; 74: 30-2.

2. Bozzetti F, Bonfanti G, Morabito A, Bufalino R, Menotti $\mathrm{V}$, Andreola S, et al. A multifactorial approach for the prognosis of patients with carcinoma of the stomach after curative resection. Surg Gynecol Obstet 1986; 162: 229-34.

3. Maruyama K. The most important prognostic factors for gastric cancer patients. Scand J Gastroenterol 1987; 22: 63-8.

4. Adachi Y, Ogawa Y, Sasaki Y, Yukaya H, Mori M, Sugimachi K. A clinicopathologic study of gastric carcinoma with reference to age of patients. J Clin Gastroenterol 1994; 18: 287-90.

5. Siewert JR, Bottcher K, Stein HJ, Roder JD. Relevant prognostic factors in gastric cancer: ten-year results of the German Gastric Cancer Study. Ann Surg 1998; 228: 449-61.

6. Lee CC, Wu CW, Lo SS, Chen JH, Li AF, Hsieh MC, et al. Survival predictors in patients with node-negative gastric carcinoma. J Gastroenterol Hepatol 2007; 22: 1014-8.

7. Wu CW, Hsieh MC, Lo SS, Tsay SH, Li AF, Lui WY, et al. Prognostic indicators in patients with gastric cancer after resection. Dig Dis Sci 1997; 42: 1265-9.

8. Baiocchi GL, Tiberio GA, Minicozzi AM, Morgagni P, Marrelli D, Bruno L, et al. A multicentric Western analysis of prognostic factors in advanced, node-negative gastric cancer patients. Ann Surg 2010; 252: 70-3.
9. Hyung WJ, Lee JH, Choi SH, Min JS, Noh SH. Prognostic impact of lymphatic and/or blood vessel invasion in patients with node-negative advanced gastric cancer. Ann Surg Oncol 2002; 9: 562-7.

10. del Casar JM, Corte MD, Álvarez A, García I, Bongera M, González LO, et al. Lymphatic and/or blood vessel invasion in gastric cancer: relationship with clinicopathological parameters, biological factors and prognostic significance. J Cancer Res Clin Oncol 2008; 134: 153-61.

11. Kim DY, Seo KW, Joo JK, Park YK, Ryu SY, Kim HR, et al. Prognostic factors in patients with node-negative gastric carcinoma: a comparison with node-positive gastric carcinoma. World J Gastroenterol 2006; 12: 1182-6.

12. Wu CW, Hsieh MC, Lo SS, Tsay SH, Lui WY, P'eng FK. Relation of number of positive lymph nodes to the prognosis of patients with primary gastric adenocarcinoma. Gut 1996; 38: 525-7.

13. Kooby DA, Suriawinata A, Klimstra DS, Brennan MF, Karpeh MS. Biologic predictors of survival in nodenegative gastric cancer. Ann Surg 2003; 237: 828-37.

14. Adachi Y, Mori M, Maehara Y, Kitano S, Sugimachi K. Prognostic factors of node-negative gastric carcinoma: univariate and multivariate analyses. J Am Coll Surg 1997; 184: 373-7.

15. Mori M, Sugimachi K. Clinicopathologic studies of gastric carcinoma. Semin Surg Oncol 1990; 6: 19-27.

16. Adachi Y, Oshiro T, Mori M, Maehara Y, Sugimachi K. Tumor size as a simple prognostic indicator for gastric carcinoma. Ann Surg Oncol 1997; 4: 137-40.

17. Maehara Y, Kabashima A, Koga T, Tokunaga E, Takeuchi $\mathrm{H}$, Kakeji Y, et al. Vascular invasion and potencial for tumor angiogenesis and metastasis in gastric carcinoma. Surgery 2000; 128: 408-16.

18. McCready DR, Chapman JA, Hanna WM, Kahn HJ, Yap $\mathrm{K}$, Fish EB, et al. Factors associated with local breast cancer recurrence after lumpectomy alone: postmenopausal women. Ann Surg Oncol 2000; 7: 562-7.

19. Nagakawa T, Mori K, Nakano T, Kadoya M, Kobayashi $\mathrm{H}$, Akiyama T, et al. Perineural invasion of carcinoma of the pancreas and biliary tract. Br J Surg 1993; 80: 619-21.

20. Yamaguchi R, Nagino M, Oda K, Kamiya J, Uesaka K, Nimura Y. Perineural invasion has a negative impact on survival of patients with gallbladder carcinoma. $\mathrm{Br} \mathrm{J}$ Surg 2002; 89: 1130-6.

21. Ruo L, Tickoo S, Klimstra DS, Minsky BD, Saltz L, Mazumdar $\mathrm{M}$, et al. Long-term prognostic significance of extent of rectal cancer response to preoperative radiation and chemotherapy. Ann Surg 2002; 236: 75-81.

22. Mori M, Adachi Y, Kamakura T, Ikeda Y, Maehara Y, Sugimachi K. Neural invasion in gastric carcinoma. J Clin Pathol 1995; 48: 137-42. 